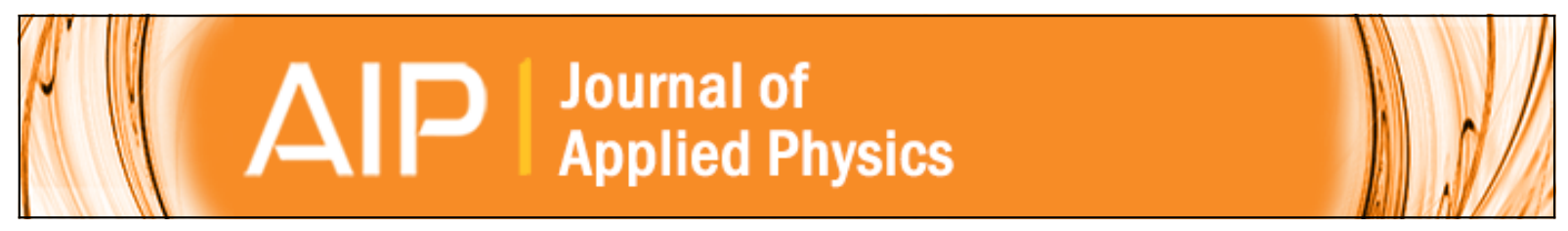

\title{
Hydrodynamic model for a vacuum arc operated with background gas: Theory and experimental validation
}

D. Grondona, H. Kelly, and F. O. Minotti

Citation: Journal of Applied Physics 99, 043304 (2006); doi: 10.1063/1.2168239

View online: http://dx.doi.org/10.1063/1.2168239

View Table of Contents: http://scitation.aip.org/content/aip/journal/jap/99/4?ver=pdfcov

Published by the AIP Publishing

\section{Articles you may be interested in}

Puzzling differences in bismuth and lead plasmas: Evidence for the significant role of neutrals in cathodic vacuum arcs

Appl. Phys. Lett. 91, 091502 (2007); 10.1063/1.2776858

Differences in the metallic plasma-neutral gas structure in a vacuum arc operated with nitrogen and argon

J. Appl. Phys. 96, 3077 (2004); 10.1063/1.1776628

Role of metallic neutrals and gaseous molecular ions in a copper cathodic arc operated with oxygen gas

J. Appl. Phys. 90, 3174 (2001); 10.1063/1.1397290

Spectral plasma temperature determination of thermionic vacuum arc in the titanium vapors

J. Vac. Sci. Technol. A 19, 757 (2001); 10.1116/1.1368839

Ion velocities in vacuum arc plasmas

J. Appl. Phys. 88, 5618 (2000); 10.1063/1.1321789

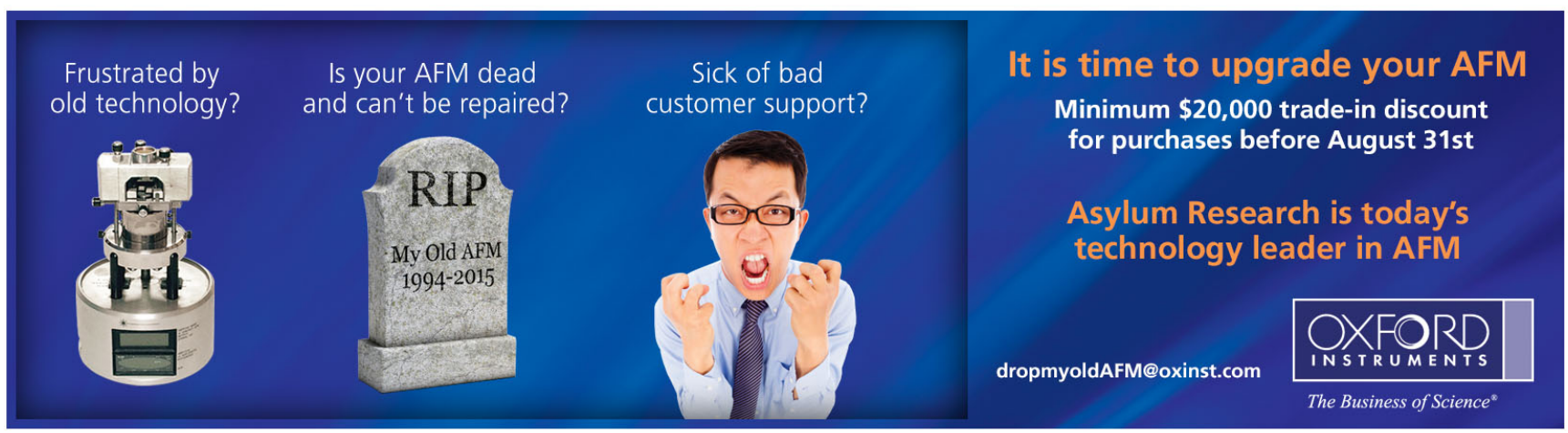




\title{
Hydrodynamic model for a vacuum arc operated with background gas: Theory and experimental validation
}

\author{
D. Grondona, ${ }^{\text {a) }}$ H. Kelly, and F. O. Minotti \\ Departamento de Física, Instituto de Física del Plasma (CONICET), Facultad de Ciencias Exactas y \\ Naturales (UBA), Ciudad Universitaria Pabello'n I, 1428 Buenos Aires, Argentina
}

(Received 15 June 2005; accepted 14 December 2005; published online 24 February 2006)

\begin{abstract}
A stationary, one-dimensional fluid model is presented to describe the interelectrode region of a nonfiltered vacuum arc operated with a background gas. The model includes the electron energy equation and the main elastic and inelastic atomic processes among metallic ions, electrons, and gas particles. To validate the model predictions an experimental study of the plasma-neutral gas structure, using a titanium (Ti) cathode and argon (Ar) as the background gas, is presented. The measured electron temperature and the experimental dependence on the pressure of neutral $\mathrm{Ti}$ and Ar spectroscopic emission lines are well reproduced, using a simple atomic model to interpret the plasma radiation emission. (C) 2006 American Institute of Physics. [DOI: 10.1063/1.2168239]
\end{abstract}

\section{INTRODUCTION}

Cathodic vacuum arcs (CVAs) are widely employed to produce coatings. ${ }^{1}$ In these devices, an intense metallic plasma jet is generated from minute regions on the cathode surface (cathode spots), with ion kinetic energies in the range of $20-120 \mathrm{eV}$, depending on the cathode material and on the charge state of the ions. ${ }^{2,3}$

In many situations, CVA complex coatings are obtained by combining metallic ions with molecules (or atoms) of a reactive gas introduced in the discharge chamber. ${ }^{4}$ Also, a nonreactive gas such as argon has been introduced to produce a pretreatment of the substrate surface that improves film adhesion ${ }^{5}$ or because of the well-known arc-stabilizing effects of Ar. ${ }^{6,7}$

When a background gas is present, a plasma-neutral gas structure is formed, where the metallic ions lose their kinetic energy through elastic and inelastic interactions with the gas particles, change their original charge state by chargeexchange processes, and are eventually lost by recombination processes. Usually, all these processes occur in the socalled interelectrode region, which is a neutral plasma zone extending from the cathode spot plasma to the anode sheath. The knowledge of this complex structure is important because the ion energy and ion flux distribution have a strong influence on the resulting film structure and properties.

Several published works have focused on plasma-gas interactions. Some of them were mainly related to the study of elastic interactions between metallic ions and neutrals. ${ }^{8-12}$ Other studies referred to inelastic interactions in the interelectrode region. From photon and ion emission studies, ${ }^{13-15}$ it was concluded that charge-exchange reactions were the main channel for metallic ion losses, in a gas pressure $(p)$ range of $10^{-6}-10^{-2} \mathrm{mbar}$. For higher gas pressure values $(p$ $>10^{-2} \mathrm{mbar}$ ), a three-body electron recombination was proposed as the main reaction for neutralizing metallic ions. ${ }^{15,16}$ Later on, ion diffusion to the chamber wall was proposed as a relevant ion-loss mechanism. ${ }^{17,18}$

${ }^{a)}$ Electronic mail: grondona@df.uba.ar
It must be noted that several theoretical models for the interelectrode region have been presented in some of the quoted works. In all of them, mass and momentum conservation equations are used to study atomic processes and ion slowing down, but energy equations are not employed. While it is true that at low $p$ values (and short distances from the cathode surface) inelastic electron collisions are very rare, at larger $p$ values exciting or ionizing collisions between electrons and heavy particles can be relevant, thus producing a depletion in the electron temperature $\left(T_{e}\right)$.

In this work, we will present a one-dimensional fluid model including the electron energy equation that is appropriate to describe the interelectrode region of a nonfiltered vacuum arc. The usual geometrical configuration of these arcs is a cylindrical cathode surrounded by another cylindrical anode of large diameter (usually the discharge chamber). In this configuration, the arc current (carried by the electrons) is distributed almost in the radial direction (thus closing the electrical circuit through the shortest anode-cathode path), while the ion emission is mainly in the forward (axial) direction.

To validate the model predictions, we present also an experimental study of the plasma-neutral gas structure in $\mathrm{Ar}$ gas. A dc, nonfiltered cathodic arc operated at a current of 90 A with a titanium cathode is employed. The geometry of the arc discharge is similar to that quoted in the previous paragraph. In addition to Langmuir probes to register $T_{e}$, optical emission spectroscopy is included as a diagnostic to register the behavior of $\mathrm{Ti}$ and $\mathrm{Ar}$ lines with the gas pressure, and the obtained results are confronted with the model predictions.

\section{EQUATIONS OF THE MODEL}

The one-dimensional (1D) stationary model simulates the axial profiles of density and velocity of different species, as well as electron temperature and axial electric field. The model is valid in the interelectrode region outside the main discharge channel and describes the plasma formed by the metallic ions ejected mainly in the axial direction from the cathode surface. It is assumed that a sufficient number of 
randomly distributed spots are present over the surface of the cathode, so that the individual plasma jets merge and form a relatively uniform plasma region beyond the cathode sheath.

If a diatomic gas is used only molecular ions are included in the model, considering that they are generated by charge exchange with metallic ions (and also by electron impact, as a small contribution, at large gas pressure values). The generation of atomic ions would require a large energy cost because of the previous dissociation step. Besides, for typical vacuum arc operating pressures the main recombination process is dissociative recombination:

$$
\mathrm{N}_{2}^{+}+e \rightarrow \mathrm{N}+\mathrm{N}^{*}
$$

where $\mathrm{N}$ represents each atom of the diatomic molecule, the asterisk denotes an excited atom, and $e$ represents an electron. At the low densities considered only metastable levels of excitation are included, as a significant density of excited atoms can only exist for these levels.

For a monoatomic gas (we explicitly use argon), ions are also mostly produced by charge exchange with metallic ions, with also some contribution of electron-impact ionization at large gas pressure values. Since a three-body recombination is not effective in the range of ion density and electron temperature considered, gas ions recombine by dissociative recombination, which in turn requires molecular ions provided by the conversion reaction

$$
\mathrm{Ar}^{+}+2 \mathrm{Ar} \rightarrow \mathrm{Ar}_{2}^{+}+\mathrm{Ar}
$$

One thus needs to consider three additional species related to the background gas in this case: atomic gas ions, diatomic gas ions, and excited atoms.

The species considered in the model are then three species of metallic ions with charge $Z_{n} e\left(Z_{n}=n\right.$, and $\left.n=1,2,3\right)$, mass $M_{m}$, density $n_{n}$, and directed axial velocity $w_{n}$; metallic atoms of density $n_{0}$ and velocity $w_{0}$; atomic gas ions of charge $e$ (multiple gas ionization is neglected in the electron temperature range considered, $\lesssim 2 \mathrm{eV}$ ), mass $M_{a}$, density $n_{a}$, and axial velocity $w_{a}$; excited atomic gas (one species for metastable level considered) with density $n_{a}^{*}$ and velocity $w_{a}^{*}$; molecular (diatomic) gas ions of charge $e$, mass $M_{g}$, density $n_{g}$, and axial velocity $w_{g}$; and neutral background gas of uniform density $N_{0}$ assumed to be at rest. Note that, for the considerations above, $n_{a}=0$ if the background gas is diatomic.

\section{A. Mass conservation equations}

The mass conservation equation for each species is written generically as

$$
\frac{d}{d z}\left(n_{s} w_{s}\right)=\left(\frac{d n_{s}}{d t}\right)_{i}-\lambda_{s} n_{s},
$$

where $\left.d n_{s} / d t\right)_{i}$ represents the rate of change of the species density due to inelastic processes, and $\lambda_{s}$ is a frequency loss of particles due to lateral diffusion. Apart from the conversion reaction and dissociative recombination, already mentioned, the other inelastic processes considered are charge exchange between each metallic ion species and the background gas, charge exchange between metallic atoms and gas ions, ionization of the gas by electron impact, excitation of gas atoms by electron impact, and the Penning reaction between a metallic atom and an excited gas atom represented by ( $M$ symbolizes the metallic atom, and $A$ the gas atom)

$$
M+A^{*} \rightarrow M^{+}+A+e .
$$

The inelastic rate of change of the metallic species density is then

$$
\begin{aligned}
& \left(\frac{d n_{1}}{d t}\right)_{i}=\sigma_{\mathrm{ce} 2} n_{2} w_{2} N_{0}-\sigma_{\mathrm{ce} 1} n_{1} w_{1} N_{0}+k_{p} n_{0} n_{a}^{*} \\
& +\sigma_{\mathrm{ce} 0} n_{0}\left(n_{a} w_{a}+n_{g} w_{g}\right), \\
& \left(\frac{d n_{2}}{d t}\right)_{i}=\sigma_{\mathrm{ce} 3} n_{3} w_{3} N_{0}-\sigma_{\mathrm{ce} 2} n_{2} w_{2} N_{0}, \\
& \left(\frac{d n_{3}}{d t}\right)_{i}=-\sigma_{\mathrm{ce} 3} n_{3} w_{3} N_{0} \\
& \left(\frac{d n_{0}}{d t}\right)_{i}=\sigma_{\mathrm{ce} 1} n_{1} w_{1} N_{0}-\sigma_{\mathrm{ce} 0} n_{0}\left(n_{a} w_{a}+n_{g} w_{g}\right)-k_{p} n_{0} n_{a}^{*},
\end{aligned}
$$

where $\sigma_{\mathrm{cen}}$ is the charge-exchange cross section of metallic ions of charge $Z_{n} e$ with the gas, $\sigma_{\mathrm{ce} 0}$ is the charge-exchange cross section of metal atoms with gas ions, and $k_{p}$ is the rate coefficient for the Penning reaction.

For the monoatomic gas ions we have

$$
\begin{aligned}
\left(\frac{d n_{a}}{d t}\right)_{i}= & S\left(k_{i} n_{e} N_{0}+\sum_{n=1}^{3} \sigma_{\mathrm{ce} n} n_{n} w_{n} N_{0}\right)-k_{\mathrm{conv}} n_{a} N_{0}^{2} \\
& +k_{i *} n_{a}^{*} n_{e}-\sigma_{\mathrm{ce} 0} n_{a} w_{a} n_{0},
\end{aligned}
$$

where $S$ is a factor of value 1 for monoatomic gas and 0 for diatomic gas, $k_{i}$ is the rate coefficient of electron-impact ionization of the gas, $k_{\text {conv }}$ is the rate coefficient of conversion to molecular ion [reaction (2)], and $k_{i^{*}}$ is the rate coefficient of electron-impact ionization of the excited atomic gas. The electron density $n_{e}$ is evaluated using local quasineutrality

$$
n_{e}=\sum_{n=1}^{3} Z_{n} n_{n}+n_{a}+n_{g} .
$$

The inelastic rate of change of molecular ions is

$$
\begin{aligned}
\left(\frac{d n_{g}}{d t}\right)_{i}= & (1-S) \\
& \times\left(k_{i} n_{e} N_{0}+\sum_{n=1}^{3} \sigma_{\mathrm{ce} n} n_{n} w_{n} N_{0}-\sigma_{\mathrm{ce} 0} n_{g} w_{g} N_{0}\right) \\
& +k_{\mathrm{conv}} n_{a} N_{0}^{2}-\beta_{\mathrm{rec}} n_{g} n_{e}
\end{aligned}
$$

where $\beta_{\text {rec }}$ is the rate coefficient of dissociative recombination [reaction (1)].

For the excited atomic gas species we have 


$$
\left(\frac{d n_{a}^{*}}{d t}\right)_{i}=k_{\mathrm{ee}} n_{e} N_{0}-k_{i *} n_{a}^{*} n_{e}-k_{p} n_{0} n_{a}^{*}+\beta_{\mathrm{rec}} n_{g} n_{e}
$$

where $k_{\mathrm{ee}}$ is the rate coefficient of electron-impact excitation of gas atoms to the metastable level considered.

\section{B. Momentum equations}

The momentum equation for each species is written generically as

$$
M_{s} w_{s} \frac{d w_{s}}{d z}=-\frac{k_{B} T_{m}}{n_{s}} \frac{d n_{s}}{d z}+Z_{s} e E-\sigma_{0 s} N_{0} w_{s}^{2} \frac{M_{s}^{2}}{M_{s}+M_{g}},
$$

where $k_{B}$ is the Boltzmann constant, $T_{s}$ is the species temperature, $E$ is the axial electric field, and $\sigma_{0 s}$ is the elastic impact cross section with the gas. In the case of the ions of the background gas we add to $\sigma_{0 s}$ the cross section of charge exchange with the gas itself, $\sigma_{\text {res }}$, as this resonant process is dominant over the elastic one. For the electrons, we neglect their inertia to write the equation that determines the electric field as

$$
e E=-\frac{k_{B} T_{e}}{n_{e}} \frac{d n_{e}}{d z} .
$$

For the neutral metallic atoms, as $Z_{0}=0$, we need to include the rate of impulse injection by neutralization of the $Z_{1}$ metallic ions as a source of momentum, so we write

$$
\begin{aligned}
M_{m} w_{0} \frac{d w_{0}}{d z}= & -\frac{k_{B} T_{m}}{n_{0}} \frac{d n_{0}}{d z}+M_{m} \frac{w_{1}}{n_{0}}\left(\frac{d n_{0}}{d t}\right)_{i} \\
& -\sigma_{00} N_{0} w_{0}^{2} \frac{M_{m}^{2}}{M_{m}+M_{g}} .
\end{aligned}
$$

\section{Electron temperature equation}

The electron temperature $T_{e}$ is determined from the energy equation for the electrons written as

$$
\frac{3}{2} w_{e} \frac{d T_{e}}{d z}=-U_{i} k_{i} N_{0}-U_{\mathrm{ee}} k_{\mathrm{ee}} N_{0}-U_{i *} k_{i *} n_{a}^{*}-\frac{3}{2} \gamma_{e} T_{e},
$$

where the $U$ 's correspond to the energy exchanged per electron in the corresponding reaction, $\gamma_{e}$ models the energy lost by thermal conduction (the lost due to lateral conduction minus the gain due to axial conduction from the cathode), and the electron fluid velocity is taken as

$$
w_{e}=\frac{1}{n_{e}}\left(\sum_{n=1}^{3} Z_{n} n_{n} w_{n}+n_{a} w_{a}+n_{g} w_{g}\right) .
$$

Of particular importance are the loss terms in Eqs. (3) and (16). They model diffusive terms of the form

$$
-\lambda_{s} n_{s} \rightarrow D_{s} \nabla_{\perp}^{2} n_{s}, \quad-\gamma_{e} T_{e} \rightarrow \kappa_{e} \nabla^{2} T_{e},
$$

where $D_{s}$ is the particle diffusion coefficient for species $s, \nabla_{\perp}^{2}$ represents the Laplacian in the plane perpendicular to the axis, and $\kappa_{e}$ is the electron thermal diffusivity (the Laplacians are evaluated at the axis position). We use the same approach as in Ref. 19 in which the radial diffusion is estimated, solving analytically the mass conservation equation with lateral diffusion without source terms to obtain an analytical density profile $n_{A}$ from which one evaluates

$$
\lambda_{s}=-\frac{D_{s}}{n_{A}} \nabla_{\perp}^{2} n_{A} .
$$

Analogously, the thermal diffusion equation is solved (this time including the axial contribution in the Laplacian) to obtain an analytical temperature profile $T_{A}$. We proceed by writing the diffusion equation as

$$
w_{e} \frac{\partial T_{A}}{\partial z}=\kappa_{e} \nabla_{\perp}^{2} T_{A}+\kappa_{e} \frac{\partial^{2} T_{A}}{\partial z^{2}}=-\lambda_{e} T_{A}+\kappa_{e} \frac{\partial^{2} T_{A}}{\partial z^{2}},
$$

where $\lambda_{e}$ is evaluated in the same way as $\lambda_{s}$, using $\kappa_{e}$ instead of $D_{s}$. For smoothly varying coefficients this equation has the correctly decaying solution of the form $T_{A} \sim \exp (-k z)$, with

$$
k=\frac{\sqrt{w_{e}^{2}+4 \kappa_{e} \lambda_{e}}-w_{e}}{2 \kappa_{e}},
$$

so that we approximate

$$
\kappa_{e} \nabla^{2} T_{A} \simeq-\left(\lambda_{e}-\kappa_{e} k^{2}\right) T_{A} \equiv-\gamma_{e} T_{A}
$$

that gives $\gamma_{e}$ in terms of $\lambda_{e}$.

\section{INTEGRATION SCHEME}

Mass continuity, momentum, and electron temperature equations are discretized using backward finite difference approximations for the spatial derivatives. Given the values of densities, axial velocities, and electron temperature at the cathode, the values along the axis are obtained, solving the explicit algebraic equations obtained from the backward spatial discretization. Velocities of the metallic ions are taken as those corresponding to the experimental values of ion energies in vacuum conditions, ${ }^{20}$ their densities are prescribed according to the experimental relative abundances, considering that they carry a given fraction, dependent on the gas pressure (see below), of the discharge current, and assuming that they are emitted from the whole surface of the cathode. Velocities and densities of the other species (other than the background gas) are taken as zero near the cathode. A fine discretization (1000 points) is employed in the first centimeter after the cathode in order to resolve correctly the region where steep variations of the densities of ionized and excited species occur. A resolution of 50 points per centimeter was found to be sufficient for the other region.

\section{EXPERIMENTAL ARRANGEMENT}

The experimental setup is shown schematically in Fig. 1. The vacuum chamber is a stainless-steel cylinder with a length of $40 \mathrm{~cm}$ and a diameter of $25 \mathrm{~cm}$, acting as the grounded anode with a water-cooling system. The cathode is a water-cooled Ti cylinder with a diameter of $5.6 \mathrm{~cm}$, surrounded by a floating shielding. The cathode is located on one end of the cylinder and is electrically connected to the negative terminal of the arc power supply.

The discharge circuit consists of a current supply (18 $\mathrm{kW}, 150 \mathrm{~A})$ in parallel with a capacitor bank $(165 \mathrm{mF})$ connected to the electrodes through a series inductor $(2.8 \mathrm{mH})$ in 


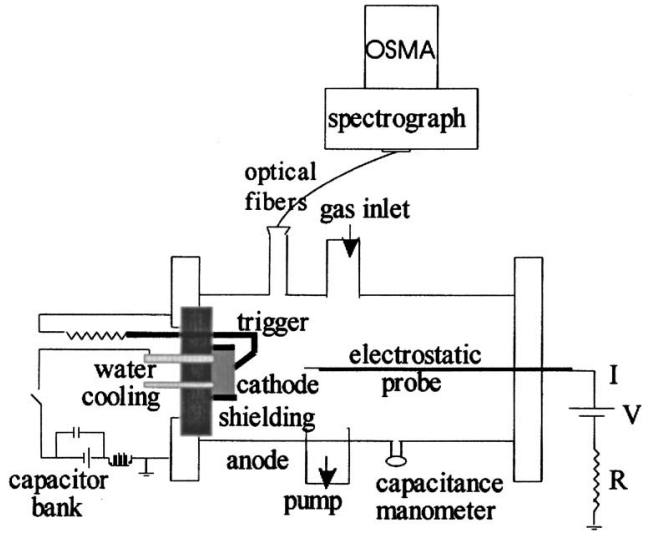

FIG. 1. Experimental setup.

order to provide the arc stability. A tungsten striker brought into contact with the cathode ignites the discharge.

The chamber is evacuated down to $10^{-5} \mathrm{mbar}$ and the $\mathrm{Ar}$ gas is introduced into the apparatus and its pressure monitored with a capacitance manometer. The arc is operated in a continuous mode with an arc current of $90 \mathrm{~A}$ and with a constant pressure ranging from $10^{-3}$ to 1 mbar.

For the spectroscopic diagnostic a circular optical window $(4 \mathrm{~cm}$ in diameter) was mounted on a tube with a length of $10 \mathrm{~cm}$ extending beyond the lateral wall of the chamber. The tube that avoids the light coming from the cathode spots enters to the detecting system (see Fig. 1). The window was located at an axial distance of $9 \mathrm{~cm}$ from the cathode surface. The light emitted by the plasma was transported from the optical window to the entrance slit of the spectrograph by optical fibers (1.5 $\mathrm{mm}$ in diameter). The spectrograph had a crossed Czerny-Turner design with an optical grating of 1200 lines $/ \mathrm{mm}$. The light from the spectrograph was intensified by a multichannel plate and detected by a photodiode array (OSMA). The wavelength calibration was made using cadmium and neon lamps, and the resulting accuracy of the detection system was $0.4 \mathrm{~nm}$, determined by the line width. The titanium and argon emission lines registered during the arc discharge were identified using published tables and databases of spectral lines. ${ }^{21,22}$

In order to determine the electron temperature, an electrostatic probe was mounted at the tip of a sliding rod, and so different cathode-probe distances $(d)$ along the axis of the chamber were obtained without opening the chamber. The distance was vaired in the range of 3-27 cm. The collecting probe electrode was a cylindrical tungsten wire of $1 \mathrm{~mm}$ in diameter and $4 \mathrm{~mm}$ long and the probe's body was wrapped with a double glass tube. The probe was biased by a power supply $( \pm 50 \mathrm{~V}, 2 \mathrm{~A})$ through a calibrated resistor. The voltage drop on the resistor determined the probe current, and the electron current collected by the probe is given as ${ }^{23}$

$$
I_{e}=I_{e 0} \exp \left[e\left(V_{p}-\varphi\right) / k T_{e}\right]
$$

where $I_{e 0}$ is the electron saturation current, $k$ is the Boltzmann constant, $V_{p}$ is the probe potential, and $\varphi$ is the plasma potential. The electron temperature was obtained from the slope of the electron branch of the probe characteristic.

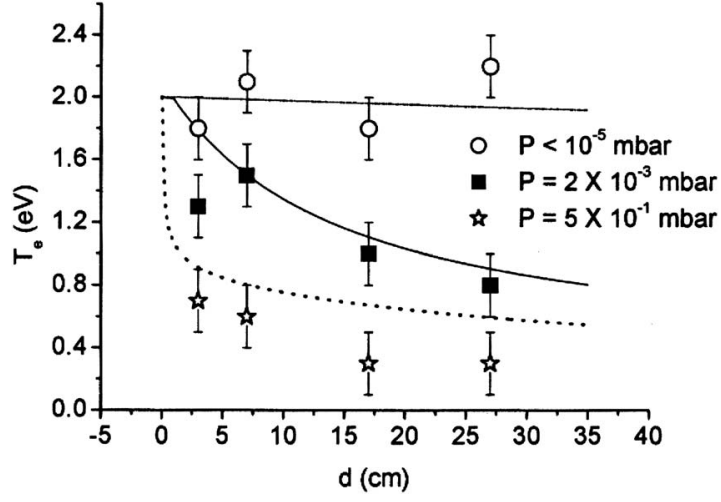

FIG. 2. Electron temperature along the chamber axis. Dots-vacuum, squares $-p=2 \times 10^{-3} \mathrm{mbar}$, and stars- $p=5 \times 10^{-1} \mathrm{mbar}$. The lines indicate the results of the theoretical model.

\section{EXPERIMENTAL RESULTS}

Figure 2 shows $T_{e}$ as a function of $d$ for vacuum ( $p$ $<10^{-5} \mathrm{mbar}$ ) and for two different values of $p$. In vacuum, the temperature is approximately constant, of value $2 \mathrm{eV}$. When gas is present, the electron temperature decreases as $d$ increases and, at a given distance, it decreases as the gas pressure increases. For $p=2 \times 10^{-3} \mathrm{mbar}, T_{e}$ is approximately $1.6 \mathrm{eV}$ at $d=3 \mathrm{~cm}$ and around $0.9 \mathrm{eV}$ at $d=27 \mathrm{~cm}$, while for $p=0.5 \mathrm{mbar}, T_{e}$ has decreased to 0.7 and $0.3 \mathrm{eV}$, for the same $d$ values. The continuous lines in the figure indicate the results of the theoretical model, which will be discussed in the next section.

The reproducibility of the registered lines obtained with the spectral diagnostic was found to be quite good. The exposure time of the detector was $0.5 \mathrm{~s}$ in all cases. The statistical dispersion in the intensity of a given line (over approximately equal to ten registers performed under similar conditions) was not larger than $10 \%$. For all the pressure range investigated, the lines of TiI and TiII were registered and, for pressure values higher than $10^{-2} \mathrm{mbar}$, also the ArI lines were observed. However, no lines of TiIII or ArII could be identified, according to the published data in spectral line tables. This is an expected result, since the charge exchange is very strong to eliminate $\mathrm{Ti}^{++}$ions even with on small amount of Ar (low $p$ values); and on the other hand, the excitation of $\mathrm{Ar}^{+}$would require larger $T_{e}$ values than those presented in Fig. 2.

The relative amplitude of the line peaks was found to be strongly dependent on the value of the Ar pressure. To study the evolution of the amplitude of a given line with $p$, and taking into account the wavelength resolution of the spectroscopic system $(\sim 0.4 \mathrm{~nm})$, we selected $\lambda=466.76 \mathrm{~nm}$ for TiI and $\lambda=811.53 \mathrm{~nm}$ for ArI. These two lines are not surrounded by other neighbor lines that could make the interpretation of the results ambiguous. The TiI line corresponds to the transition $3 d^{3} 4 s^{2}-3 d^{2}\left({ }^{3} F\right) 4 s 4 p\left({ }^{3} P^{0}\right)$ of neutral titanium, with binding energies of $2.68 \mathrm{eV}$ (upper level) and $0.021 \mathrm{eV}$ (lower level) with respect to the ground state. The ArI line considered corresponds to the transition $3 s^{2} 3 p^{5}\left({ }^{2} P^{0} 3 / 2\right) 4 s-3 s^{2} 3 p^{5}\left({ }^{2} P^{0} 3 / 2\right) 4 p^{0}$ with an upper level of energy $13.07 \mathrm{eV}$ and a lower level (metastable level) of energy $11.59 \mathrm{eV}$. The lines of TilI were not selected in this 


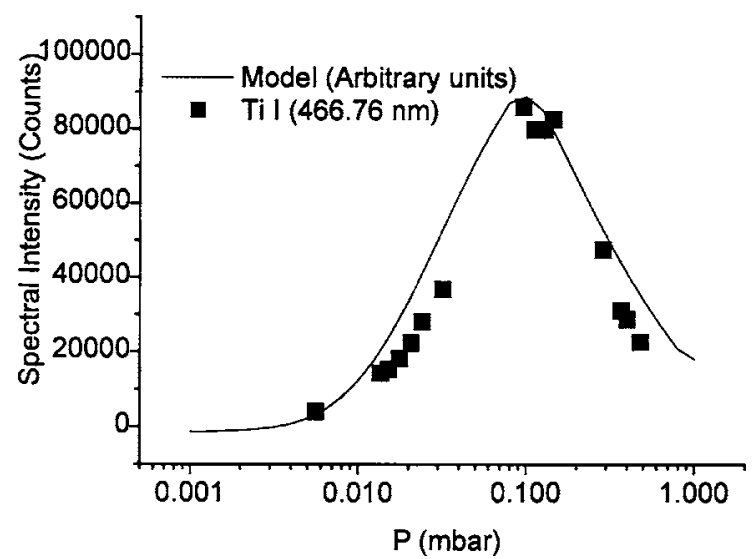

FIG. 3. Spectral intensity emitted from Ti I $(466.76 \mathrm{~nm})$ vs pressure. The lines indicate the results of the theoretical model.

study, since the intense lines of TiII (at low $p$ values) were soon overlapped by TiI or ArI lines for higher $p$ values in the spectral windows used in this study $(\lambda \approx 440-480 \mathrm{~nm}$ and $\lambda \approx 790-830 \mathrm{~nm}$ ).

In Fig. 3 the behavior of the TiI line with the Ar filling pressure is shown. The vertical axis on the left side of the figure represents the count number registered by the detector. These line intensities are defined by subtracting the background counts (around 1500 counts in this spectral region) to the peak height. The theoretical result is represented with a continuous line in the figure.

In Fig. 4 the behavior of the ArI line with the Ar filling pressure is shown. The units of the vertical axis and the meaning of the continuous line are the same as in the previous figure.

\section{INTERPRETATION OF THE RESULTS AND DISCUSSION}

Since the plasma light source is extended over the whole discharge chamber, in order to predict the pressure dependence of the intensity of different lines as observed at the fixed optical window, it is necessary to know the axial and radial distributions of the emitting particles (neutrals and ions), together with an adequate radiative model, which gives the power radiated in each plasma volume element in

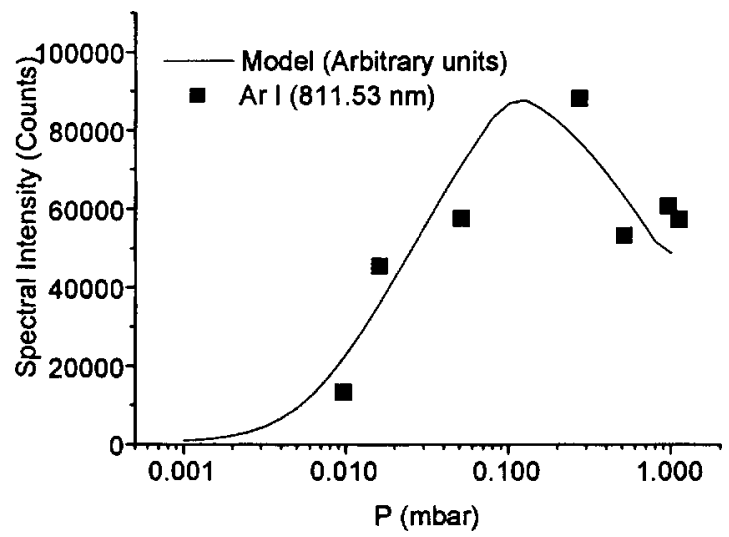

FIG. 4. Spectral intensity emitted from Ar I $(811.35 \mathrm{~nm})$ vs pressure. The lines indicate the results of the theoretical model. terms of the population of emitting particles with given excited levels (note that the lines typical of vacuum arcs correspond to the transitions from highly excited levels). Since radial density profiles and relative population of particles with particular excited levels cannot be obtained from the presented one-dimensional hydrodynamic model, a simple model of the light collected from the plasma was adopted. We have assumed that the light is emitted mostly from the axis, where the highest densities of particles exist. As for the excited states, it is assumed that the upper level is populated by electron impact from the fundamental level, and depopulated by radiative emission, in a coronalike equilibrium appropriate for the low-density plasma considered. According to this model, the power (per unit volume) of line emission for the transition from level $n$ to $m$ is modeled as ${ }^{24}$

$$
\begin{aligned}
I_{n m}(z)= & C\langle g(n, 0)\rangle \frac{n_{e}(z) n_{i 0}(z) f_{n m} g_{0}}{g_{m} T_{e}^{1 / 2}(z)} \\
& \times\left(\frac{\Delta E_{n m}}{\Delta E_{n 0}}\right)^{3} \exp \left[-\frac{\Delta E_{n m}}{T_{e}(z)}\right],
\end{aligned}
$$

where $C$ is a constant of value $2.56 \times 10^{-24} \mathrm{~W} \mathrm{~cm}^{3} \mathrm{eV}^{1 / 2}, n_{e}$ is the density of electrons, and $n_{i 0}$ that of emitting ions or atoms in the fundamental level (or metastable level), to which the subindex 0 refers. The energy differences between levels $n$ and $m, \Delta E_{n m}$, and the electron temperature are given in $\mathrm{eV}$. The thermal-averaged Gaunt factor $\langle g(n, 0)\rangle$ is taken as 0.2 for ions and 1 for neutrals. ${ }^{24}$ The other magnitudes, taken from Ref. 21 , are the oscillator strength $f_{n m}$, and $g_{l}$, the statistical weight of level $l$. For the TiI line we have $f_{n m}$ $=9.16 \times 10^{-3}, g_{0}=5, g_{m}=7, \Delta E_{n 0}=2.68 \mathrm{eV}$, and $\Delta E_{n m}=2.66$ $\mathrm{eV}$. For the ArI line the values are $f_{n m}=0.458, g_{0}=g_{m}=5$, and $\Delta E_{n 0}=\Delta E_{n m}=1.48 \mathrm{eV}$, where the lower level considered is a metastable one, and so the density $n_{i 0}(z)$ is that given in the model as $n_{a}^{*}(z)$.

The light collected at the window is taken as proportional to the local power (per unit volume) emitted by the line, times the solid angle displayed by the window entrance at the point of emission. With this simple model the power measured at the window $I_{w}$ can be expressed as proportional to

$$
I_{w} \propto \int_{0}^{z_{c}} \frac{I_{n m}(z) d z}{\left[R_{c}^{2}+\left(z-z_{w}\right)^{2}\right]^{3 / 2}},
$$

where $R_{c}$ is the radius of the chamber, $z_{w}$ is the axial position of the window, and $z$ is the generic point on the axis where the line intensity per unit volume (in $\mathrm{W} \mathrm{cm}^{-3}$ units) is $I_{n m}(z)$. The integral is taken along the axis from the position of the cathode $(z=0)$ to the chamber length $\left(z=z_{c}\right)$. Expression (19) is only proportional to the measured power, the constant of proportionality being dependent on geometrical factors such as the window surface and chamber radius, and also to the radial profile of the species considered. As mentioned above, the latter cannot be estimated with sufficient precision with the one-dimensional model, so that we have left the proportionality constant unspecified and used Eq. (19) in arbitrary units. For the same reason the measured intensities (in number of counts) were not converted to absolute values (W), 
although care was taken to keep the same acquisition time in all measurements in order that the proportionality between number of counts and measured power be the same in all cases.

The numerical code gives the density of electrons and of different species of atoms and ions, and the electron temperature (the density obtained for a given species includes all different excited states, but in the assumed statistical equilibrium this density practically coincides with that of the fundamental level). The results from the code can be directly used to evaluate $I_{n m}(z)$ at each $z$ position along the axis and so to evaluate expression (19).

The theoretical model was run for values of the arc parameters corresponding to our experimental situation. The ionic current was taken as $8 \%$ of the $90 \mathrm{~A}$ main arc current for pressures up to 0.1 mbar. $^{25}$ For higher pressures we considered an exponential decrease with the pressure of this fraction, down to $3 \%$ at $1 \mathrm{mbar}$, to match the observed decrease in the cathode erosion rate. ${ }^{26}$ The fractions of Ti ions were taken as 0.11 for the $z=+1,0.77$ for the $z=+2$, and 0.12 for the $z=+3$ ions. ${ }^{27}$ It was also assumed that each metallic ion carried out an average kinetic energy of $122 \mathrm{eV}$ at the cathode surface. ${ }^{20}$ The electron temperature was chosen to be $2 \mathrm{eV}$ at the cathode surface, in agreement with the experimental values in vacuum (see Fig. 2), the temperature of metallic ions and atoms was assumed to be $0.3 \mathrm{eV},{ }^{28}$ and the gas temperature was $0.025 \mathrm{eV}^{8}$

The rate coefficients for the different processes were taken from Refs. 29-33 and are given as elastic interaction between metallic particles and gaseous ions and gas molecules, $\sigma_{0 s}=2 \times 10^{-15} \mathrm{~cm}^{2}$, charge-exchange processes between metallic ions and the gas atoms, $\sigma_{\mathrm{ce} 1}=10^{-16} \mathrm{~cm}^{2}$, $\sigma_{\mathrm{ce} 2}=2 \times 10^{-16} \mathrm{~cm}^{2}, \sigma_{\mathrm{ce} 3}=3 \times 10^{-16} \mathrm{~cm}^{2}, \quad$ charge-exchange processes between metallic neutral and the gas ions, $\sigma_{\text {ce } 0}$ $=10^{-15} \mathrm{~cm}^{2}$, resonant charge-exchange processes between gas atoms and gas ions $\sigma_{\text {res }}=7 \times 10^{-15} \mathrm{~cm}^{2}$, electron-impact ionization of $\mathrm{Ar}$ gas, $k_{i}=6.69 \times 10^{-10} C_{i 0} T e^{1 / 2}\left(U_{\text {ion }}+2 T e\right)$ $\times \exp \left(-U_{\text {ion }} / T e\right) \mathrm{cm}^{3} \mathrm{~s}^{-1} \quad$ (where $C_{i 0}=2 \times 10^{-17} \mathrm{~cm}^{2} \mathrm{eV}^{-1}$ and $U_{\text {ion }}=15.8 \mathrm{eV}$ ), Penning ionization of Ti by excited Ar, $k_{p}=9 \times 10^{-15} \mathrm{~cm}^{2} \times w_{0}$, conversion reaction for Ar gas into molecular gas, $k_{\mathrm{conv}}=5.5 \times 10^{-31} \mathrm{~cm}^{6} \mathrm{~s}^{-1}$, dissociative recombination of molecular gas ions, $\beta_{\text {rec }}=10^{-7} \mathrm{~cm}^{3} \mathrm{~s}^{-1}$, excitation of $\mathrm{Ar}$ by electron impact, $k_{\mathrm{ee}}=2.2 \times 10^{-8} \mathrm{Te}^{1 / 2}$ $\times \exp (-12.4 / \mathrm{Te}) \mathrm{cm}^{3} \mathrm{~s}^{-1}$, and ionization of excited Ar by electron impact, $k_{i^{*}}=2.1 \times 10^{-7} T e^{1 / 2} \exp (-5.3 / T e)$.

It can be seen from Fig. 2 that the electron temperatures predicted by the model are in fairly good agreement with those obtained in the experiment. The fall in $T_{e}$ at relatively high $p$ values is mainly due to electron energy losses in excitation and ionization processes.

The behavior of the electron temperature shown in Fig. 2 can be understood by analyzing the relative weight of the terms in the right-hand side of Eq. (16). At low-pressure values the excitation and ionization terms (the first three terms) are very small, and the temperature evolution is controlled by the last term, the thermal conduction term. At larger $p$ values the inelastic terms (mainly excitation of $\mathrm{Ar}$ from the ground state) dominate over conduction close to the cathode surface, thus producing a marked decrease of $T_{e}$.
This reduction of temperature decreases the rate of inelastic losses, and as one moves away from the cathode (between 3 and $5 \mathrm{~cm}$ from the cathode surface in the pressure range investigated) the thermal conduction term dominates again. As a result, above $\sim 10^{-2}$ mbar, and farther than $\sim 5 \mathrm{~cm}$ from the cathode, the electron temperature has already decreased to a value of $\simeq 0.4 \mathrm{eV}$ and stays approximately constant.

From Figs. 3 and 4, it can be seen that the adjustment of the line intensities with the results predicted by Eq. (19) is quite reasonable, in spite of the simplifying assumptions involved in the theoretical model and in the radiative processes that originate the line emission.

Concerning again Fig. 3, the observed increase in the intensity of the TiI line with $p$ can be attributed to a strong charge exchange of $\mathrm{Ti}$ ions that generate neutral $\mathrm{Ti}$ (at intermediate $p$ values) together with a decrease of diffusion losses at high $p$. For large pressure values, however, the line intensity decreases because the number of Ti atoms decreases due to the reduction in the erosion rate, while $T_{e}$ remains practically constant.

The profile of the intensity of the ArI line, presented in Fig. 4, can be understood considering that this line is excited by electron impact, hence it grows with the Ar density and $T_{e}$. At high $p$ values, for which $T_{e}$ remains practically constant, the line intensity decreases because the electron density decreases due to the reduced erosion rates.

Note that the absence of TiIII lines (also found in other works), in spite of the fact that $z=+2$ is the most abundant species for a $\mathrm{Ti}$ cathode, is a consequence of the strong charge exchange of these ions with neutral Ar. The same reason explains the absence of ArII lines $\left(\mathrm{Ar}^{+}\right.$ions are also destroyed by conversion reactions, producing $\mathrm{Ar}_{2}^{+}$, which are in turn destroyed by dissociative recombination).

\section{FINAL REMARKS}

We have presented a hydrodynamic model valid for the interelectrode region of a vacuum arc operated with background gases. Besides the usual mass conservation and momentum equations, the model includes an electron energy equation with source terms giving account for excitation and ionization of neutrals and electron energy losses by thermal conduction. For typical vacuum arc operating pressure conditions, it has been found that the thermal conduction term plays an important role in controlling the electron temperature. The predictions of the model have been compared with experimental data of electron temperatures obtained at different Ar gas pressures, and a fairly good agreement has been obtained. Also, experimental pressure profiles of TiI and ArI lines have been reasonably adjusted by the model predictions, in spite of the relatively crude atomic model adopted to interpret the plasma radiation emission.

\section{ACKNOWLEDGMENTS}

This work was supported by grants from the Buenos Aires University (X 111 and X 106), the CONICET (PIP 02239), and the ANPCyT (ID03-09491). D. G., H. K., and F. O. M. are members of the CONICET. 
${ }^{1}$ R. L. Boxman and S. Goldsmith, IEEE Trans. Plasma Sci. 17, 705 (1989) ${ }^{2}$ J. Kutzner and H. C. Miller, IEEE Trans. Plasma Sci. 17, 688 (1989).

${ }^{3}$ I. G. Brown, Rev. Sci. Instrum. 65, 3061 (1994).

${ }^{4}$ H. Randhawa, Thin Solid Films 167, 175 (1988).

${ }^{5}$ M. K. Puchert, C. A. Davis, D. R. MacKenzie, and B. W. James, J. Vac. Sci. Technol. A 10, 3493 (1992).

${ }^{6}$ X. H. Ji, S. P. Lau, G. Q. Yu, W. H. Zhong, Y. G. Wang, and B. K. Tay, J. Phys. D 36, 2543 (2003).

${ }^{7}$ J. Cyviené, M. Laurikaitis, and J. Dudonis, Mater. Sci. Eng., B 118, 238 (2005).

${ }^{8}$ C. W. Kimblin, J. Appl. Phys. 45, 5235 (1974).

${ }^{9}$ J. L. Meunier and M. G. Drouet, IEEE Trans. Plasma Sci. 15, 515 (1987)

${ }^{10}$ R. L. Boxman and S. Goldsmith, IEEE Trans. Plasma Sci. 18, 231 (1990).

${ }^{11}$ H. Kelly, A. Márquez, and F. O. Minotti, IEEE Trans. Plasma Sci. 26, 1322 (1998).

${ }^{12}$ E. Gidalevich, S. Goldsmith, and R. L. Boxman, J. Phys. D 33, 2598 (2000).

${ }^{13}$ I. I. Demidenko, N. S. Lomino, V. D. Ovcharenko, V. G. Padalka, and G. N. Poliakova, Sov. Phys. Tech. Phys. 22, 895 (1981).

${ }^{14}$ P. J. Martin, D. R. McKenzie, R. P. Netterfield, P. Swift, S. W. Filipczuk, K. H. Muller, C. G. Pacey, and B. James, Thin Solid Films 153, 91 (1987).

${ }^{15}$ C. Bergman, Surf. Coat. Technol. 36, 243 (1988).

${ }^{16}$ M. Sakaki and T. Sakakibara, IEEE Trans. Plasma Sci. 22, 1049 (1994).

${ }^{17}$ M. M. M. Bilek, P. J. Martin, and D. R. McKenzie, J. Appl. Phys. 83, 2965 (1998).

${ }^{18}$ H. Kelly, A. Lepone, and A. Márquez, J. Appl. Phys. 89, 1567 (2001).
${ }^{19}$ D. Grondona, A. Márquez, F. Minotti, and H. Kelly, J. Appl. Phys. 96, 3077 (2004).

${ }^{20}$ G. Yu. Yushkov, A. Anders, E. M. Oks, and I. G. Brown, J. Appl. Phys. 88, 5618 (2000).

${ }^{21}$ NIST atomic spectra database (online). Available: http://physics.nist.gov/ cgi-bin/atdata

${ }^{22}$ J. Reader and C. H. Corliss Handbook of Chemistry and Physics, 78th ed. (CRC, Boca Raton. FL, 1997)

${ }^{23}$ L. Schott, Plasma Diagnostics (American Institute of Physics, New York, 1995).

${ }^{24}$ W. L. Wiese and G. A. Martin, AIP 50th Anniversary Physics Vade Mecum (American Institute of Physics, New York, 1981).

${ }^{25}$ C. W. Kimblin, J. Appl. Phys. 44, 3074 (1973).

${ }^{26}$ V. N. Zhitomirsky, O. Kiniot, B. Alterkop, R. L. Boxman, and S. Goldsmith, Surf. Coat. Technol. 263, 86 (1996).

${ }^{27}$ I. G. Brown, A. Anders, S. Anders, M. R. Dickinson, R. A. MacGill, and E. M. Oks, Surf. Coat. Technol. 84, 550 (1996).

${ }^{28}$ F. Minotti, H. Kelly, and A. Lepone, Plasma Sources Sci. Technol. 11, 294 (2002).

${ }^{29}$ L. B. Loeb, Basic Processes of Gaseous Electronics (California University Press, Berkeley, 1955).

${ }^{30}$ Y. P. Raizer, Gas Discharge Physics (Springer, Berlin, 1991), chap. 4.

${ }^{31}$ L. A. Riseberg, W. F. Parks, and L. D. Schearer, Phys. Rev. A 8, 1962 (1973).

${ }^{32}$ A. Lepone and H. Kelly, J. Phys. D 34, 3043 (2001).

${ }^{33}$ K. Tao, D. Mao, and J. Hopwood, J. Appl. Phys. 91, 4040 (2002). 